\title{
How much is a coin worth: acute pancreatitis, suspected pancreatic cancer, and a Whipple surgery caused by ingestion of a coin?
}

\author{
Ege Altan, Taylan Kav \\ Department of Gastroenterology, School of Medicine, Hacettepe University, Ankara, Turkey
}

Gastroenterology Rev 2021; 16 (4): 390-391

DOI: https://doi.org/10.5114/pg.2021.111767

\begin{abstract}
Address for correspondence: Taylan Kav, Department of Gastroenterology, School of Medicine, Hacettepe University, Ankara, Turkey, phone: +90 3123051712, e-mail: tkav@hacettepe.edu.tr
\end{abstract}

Acute pancreatitis caused by the ingestion of foreign objects is rarely reported in the literature. Oedema in the duodenal papilla, obstruction of the Wirsung canal, and increased reflux of the duodenal contents into the pancreas have been held responsible in these cases [1-3]. Acute pancreatitis can sometimes mimic pancreas cancer [4]. However, we have seen no other cases in the literature in which a foreign body, a coin, caused acute pancreatitis that mimiced a tumour around the proximal common bile duct.

A 74-year-old patient presented to the emergency room (ER) with abdominal pain and jaundice. His blood workup revealed a threefold increase in his cholestatic enzymes, a total bilirubin level increase of $7 \mathrm{mg} / \mathrm{dl}$ with direct bilirubin at $4 \mathrm{mg} / \mathrm{dl}$, and a normal amylase level. Abdominal ultrasound revealed dilated bile ducts, and computed tomography (CT) and magnetic resonance cholangiopancreatography (MRCP) were significant for a metallic foreign body in the duodenum as well as a $1.5 \mathrm{~cm}$ mass in the papillary region (Figures 1, 2). Although the patient denied any foreign body ingestion that he could remember, an upper endoscopy revealed a coin, one Turkish lira to be precise, in the second segment of the duodenum, and after coin extraction with a basket, circumferential fibrosis in the third segment (Figures 3, 4). Biopsies were taken from the fibrotic appearing peripapillary region, but as his CA 19-9 count was relatively high at $212 \mathrm{pg} / \mathrm{ml}$, the general surgery department performed a Whipple operation for a suspected ampullary tumour. Pathology of the surgical specimen revealed acute pancreatitis, peripancreatic fat necrosis, fibrosis, and chronic inflammation of the duodenum. The patient was discharged 1 week after surgery.

One other case was published in 2015 that describes a patient who presented with acute pancreatitis due to coin ingestion. In that case, the coin that was extracted had not been in circulation since 1975 . The imaging results were also suspicious for a papillary tumour, but surgery was not performed and follow-up was uneventful after the extraction of the coin [1]. Although the coin in our case was minted in 2013, it is impossible to be sure when it was ingested.

We believe there are 2 possible explanations for such an occurrence: first, the fibrosis caused by a long-standing foreign object may have resulted in acute pancreatitis; secondly, maybe a more sound explanation, the patient had chronic pancreatitis that might have led to changes mimicking a periampullary tumour, causing duodenal stenosis, which did not allow the coin to pass the duodenum, sticking instead in the periampullary region and giving rise to exacerbation of undiagnosed chronic pancreatitis and cholestasis. We would like to remind the readers of the possibility that foreign objects in the duodenum may exacerbate inflammation or may cause fibrosis mimicking periampullary tumours, and careful evaluation and follow-up may be a better option than urgent surgery in these patients.

\section{Conflict of interest}

The authors declare no conflict of interest. 


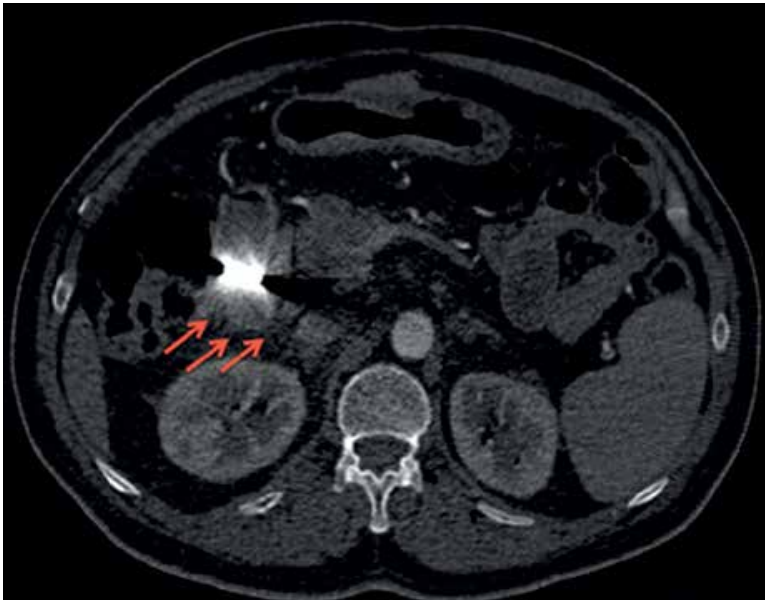

Figure 1. Metallic object in the duodenum and periampullary mass (arrows)

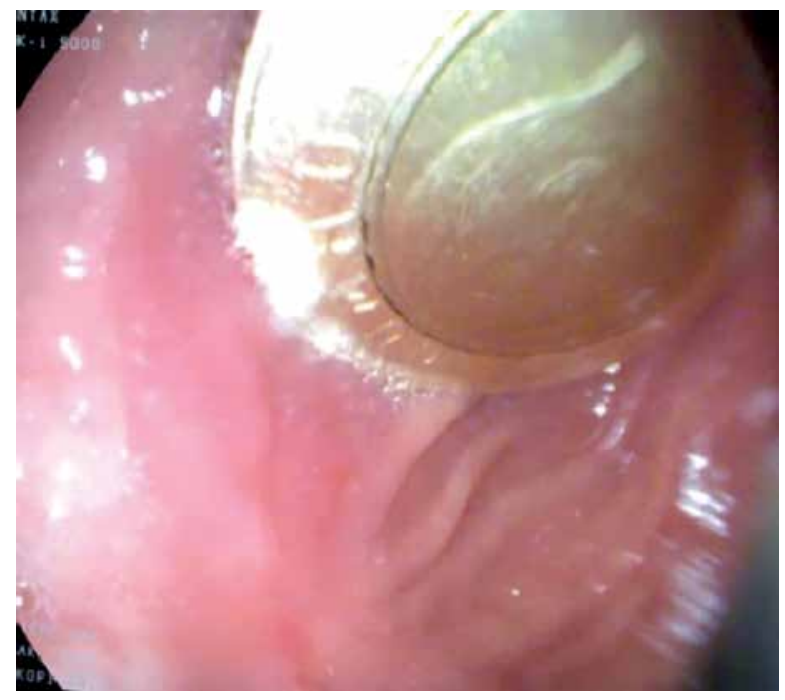

Figure 3. Coin in the duodenum

\section{References}

1. Ismail I, Mudge DW. Pancreatic and biliary obstruction years after retention of a swallowed coin in a duodenal diverticulum: a case report. J Med Case Rep 2015; 9: 144.

2. Casas J, Suarez G, Jimenez S, Verona R. Acute necrotizing pancreatitis by a foreign body. First worldwide report secondary to a match impactation. Rev Gastroenterol Peru 2010; 30: 216-9.

3. Rezgui-Marhoul L, Said W, Askri A, et al. Acute pancreatitis due to a metallic foreign body. Gastroenterol Clin Biol 2004; 28: 299-300.

4. Calculli L, Festi D, Pezzilli R. Enlarged pancreas: not always a cancer. Hepatobiliary Pancreat Dis Int 2015; 14: 107-8.

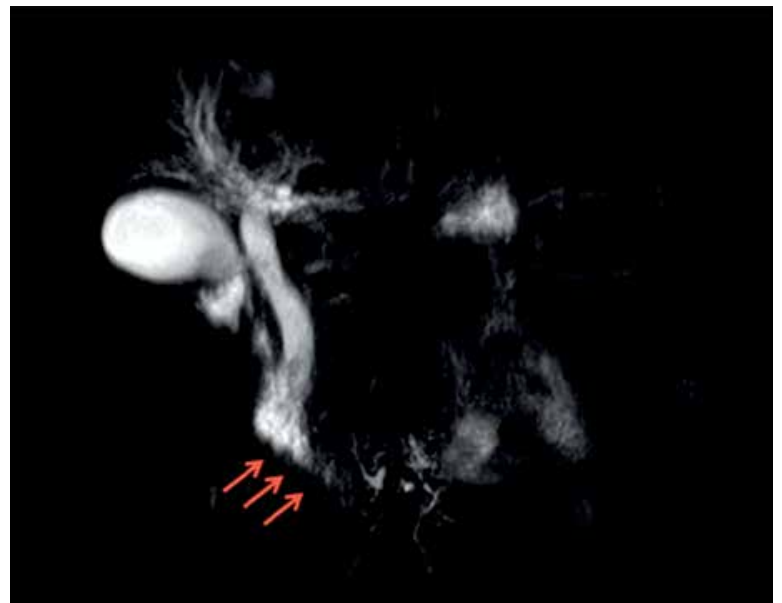

Figure 2. Magnetic resonance cholangiopancreatography suggestive of a periampullary tumour

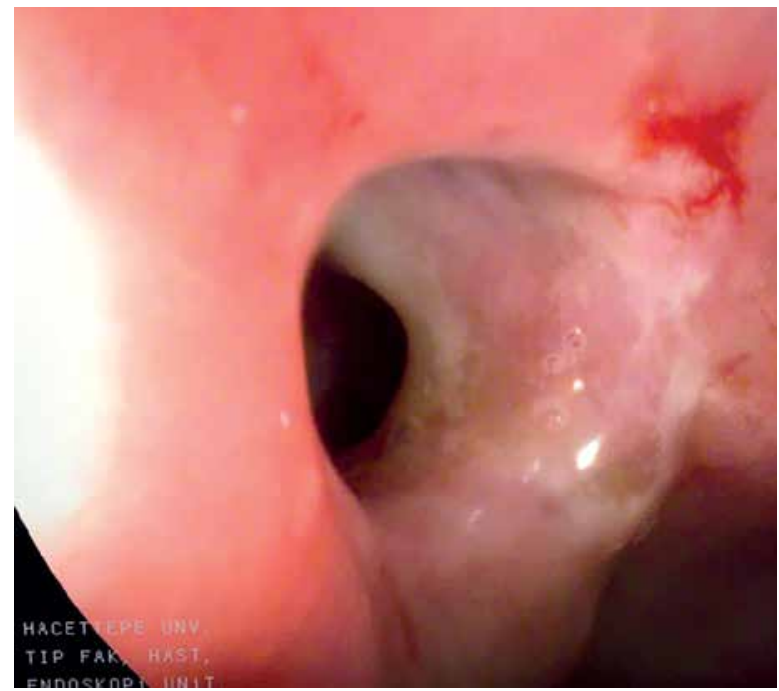

Figure 4. Duodenal stenosis revealed after coin extraction

Received: 3.10.2020

Accepted: 10.03.2021 